\title{
On the Solution to the Separated Equation in the 3-Particle Calogero-Moser Problem
}

\author{
Natalia Inozemtseva ${ }^{1}$, Jaroslav Dittrich ${ }^{2}$, Vladimir Inozemtsev ${ }^{3}$ \\ ${ }^{1}$ University “DUBNA”, Dubna, Russia \\ ${ }^{2}$ Nuclear Physics Institute CAS, Řež, Czech Republic \\ ${ }^{3}$ BLTP JINR, Dubna, Russia \\ Email: nginozv@mail.ru, dittrich@ujf.cas.cz,inozv@theor.jinr.ru
}

How to cite this paper: Inozemtseva, N. Dittrich, J. and Inozemtsev, V. (2018) On the Solution to the Separated Equation in the 3-Particle Calogero-Moser Problem. Advances in Pure Mathematics, 8, 266-271. https://doi.org/10.4236/apm.2018.83014

Received: January 15, 2018

Accepted: March 12, 2018

Published: March 15, 2018

Copyright (C) 2018 by authors and Scientific Research Publishing Inc. This work is licensed under the Creative Commons Attribution International License (CC BY 4.0).

http://creativecommons.org/licenses/by/4.0/

\begin{abstract}
We propose the exact solution of the equation in separated variable which appears in the process of constructing solutions to the quantum Calogero-Moser three-particle problem with elliptic two-particle potential $g(g-1) \wp(q)$. This solution is found for special values of coupling constants $g \in \mathbb{Z}, g>1$. It can be used for solving three-particle Calogero-Moser problem under the appropriate boundary conditions.
\end{abstract}

\section{Keywords}

Calogero-Moser Model, 3-Particle Problem, Separation of Variables

\section{Introduction}

The problem of finding solutions to quantum integrable finite-dimensional systems in many cases still remains unsolved. The empirical constructions of such solutions were important at early stages of the development of the theory of these systems and lead to many important results being applied to trigonometric Calogero-Sutherland-Moser systems with the Hamiltonian of the form

$$
H=\sum_{j=1}^{N} \frac{p_{j}^{2}}{2}+g(g-1) \sum_{j>k}^{N} V\left(q_{j}-q_{k}\right), \quad p_{j}=-i \frac{\partial}{\partial q_{j}},
$$

for $N$ particles in one dimension with the two-body potential given by

$$
V(q)=a^{2}(\sin (a q))^{-2} .
$$

The coupling constant $g$ is supposed to be real and chosen as $g>1$.

It turned out [1] [2] that the ground-state wave function of the trigonometric model is of factorized form and the wave functions of all the excitations can be 
written as products of this function and multivariable Jack polynomials [3]. These results were also extended to more complicated cases with interaction terms modified by the introduction of more general root systems [3].

The quantum elliptic many-particle problem which also has been proven to be integrable [4] [5] is till now quite far from being solved completely. It has two-particle interaction potential of the form

$$
V(q)=\wp(q)
$$

where $\wp(q)$ is the Weierstrass elliptic function with two periods $\omega_{1,2}$ which do not lie on the line in complex plane. The hermiticity of the Hamiltonian implies $\omega_{1} \in \mathbb{R}, i \omega_{2} \in \mathbb{R}$. The trigonometric case (2) corresponds to infinite complex period.

In the simplest case of $N=2$, the eigenvalue problem for the Hamiltonian (1), (3) is just the well-known Lamé equation. For general coupling constants $g$, its solutions have a branch point at the origin and their expression through the known transcendental functions is not known. However, there is an exception: for integer values of $g$, Hermite found that the solutions are expressed in terms of an exponent and quasiperiodic Weierstrass $\sigma$ functions [6]. The reason for the existence of such a solution is in fact based on its "good" analytic properties in a complex plane of the variable $q$ : at integer $g$ there is no branch points and the only singularity is a pole at $q=0$ up to the quasiperiodicity.

This fact inspired the authors in the paper [7] to consider the case of general $N>2$ and $g \in \mathbb{Z}$. It has been proved that the double quasiperiodic solutions for many-particle wave functions are still expressed in terms of the Weierstrass $\sigma$ functions but the procedure of finding them is rather complicated. They were able to find it explicitly only for $N=3, g=2$. In [8], these solutions have been presented analytically for arbitrary $N>2$ and $g \geq 2$, also in overcomplicated form requiring many nontrivial operations to their explicit writing. As for arbitrary real $g>1$ the solution of the eigenproblem for the elliptic case was constructed by the perturbation theory in the form of infinite series [9].

However, there is another approach to finding the solutions for the dynamics of integrable systems, namely separation of variables. It is well known in its simple form using purely co-ordinate transformations. As for the elliptic Calogero-Moser systems, simple forms do not work but separation still takes place as it was proposed in [10] for 3-particle case at arbitrary values of coupling constant $g$. The separation of variables occurs after transformation corresponding to a classical canonical transformation of phase space variables mixing coordinates and momenta. The transformation is realized as an integral transform of the wave function in the quantum case. The original two-dimensional problem has been reduced to one-dimensional one and the process of finding the eigenfunction contains investigation of the solution to a third order ordinary differential equation [10], 


$$
\begin{aligned}
& \psi^{\prime \prime \prime}(x)-i h_{1} \psi^{\prime \prime}(x)-\left[h_{2}+3 g(g-1) \wp(x)\right] \psi^{\prime}(x) \\
& +\left[i h_{3}+i g(g-1) h_{1} \wp(x)+g(g-1)(g-2) \wp^{\prime}(x)\right] \psi(x)=0,
\end{aligned}
$$

where $h_{1}, h_{2}, h_{3}$ are constants (the values of the integrals of motion).

The aim of this paper is to find the explicit solutions to Equation (4). We shall show below that for integer values of $g, g \geq 2$ they may be obtained via the solution to the system of $g$ usual transcendental equations.

\section{Finding the Solution}

It should be noted at first that the coefficients in (4) are double periodic functions of $x$. Hence one try to seek the particular solution as double quasiperiodic function. The second observation concerns possible singularities of this solution. Since $\wp(x)$ has double pole at $x=0$ and it is analytic at the other points of torus $\mathbb{T}=\mathbb{C} /\left(\mathbb{Z} \omega_{1}+\mathbb{Z} \omega_{2}\right)$, all the singularities of $\psi(x)$ in $\mathbb{T}$ must be also located at $x=0$. The assumption

$$
\psi(x) \sim x^{\rho}, \quad \rho<0
$$

at $x \rightarrow 0$ gives the result

$$
\psi(x) \sim x^{1-g}
$$

as the leading singularity, taking the most singular possibility only. For non-integer $g$, this is a branch point and there is no simple ansatz to the solution of (4).

The situation is changed drastically if $g \in \mathbb{Z}, g>1$. In this case, the leading singularity of $\psi(x)$ is a pole of the order $g-1$ and there are no branch points. Combining this property with double quasiperiodicity allows one to write down the Hermite-like ansatz for the possible solution to (4)

$$
\psi(x)=A \exp (\gamma x) \sigma(x)^{1-g} \prod_{s=1}^{g-1} \sigma\left(x+\lambda_{s}\right),
$$

where $A$ is inessential normalization constant, $\gamma$ and $\left\{\lambda_{s}\right\}$ are parameters which have to be determined, and $\sigma(x)$ is the Weierstrass sigma function. It is connected with $\wp(x)$ by the relations

$$
\frac{\mathrm{d}}{\mathrm{d} x} \log \sigma(x)=\zeta(x), \quad \frac{\mathrm{d}}{\mathrm{d} x} \zeta(x)=-\wp(x),
$$

where $\zeta(x)$ is the Weierstrass $\zeta$ function with the property

$$
\zeta(x)=x^{-1}+O\left(x^{3}\right), \quad x \rightarrow 0 .
$$

We assume that all $\lambda_{s}$ are mutually different for $s=1, \cdots, g-1$ and different from 0 in $\mathbb{T}$.

By consecutive differentiations of (5), one finds

$$
\frac{\psi^{\prime}}{\psi}=\gamma+\sum_{s=1}^{g-1} \zeta\left(x+\lambda_{s}\right)-(g-1) \zeta(x)
$$




$$
\begin{gathered}
\frac{\psi^{\prime \prime}}{\psi}=\left[\gamma+\sum_{s=1}^{g-1} \zeta\left(x+\lambda_{s}\right)-(g-1) \zeta(x)\right]^{2}-\sum_{s=1}^{g-1} \wp\left(x+\lambda_{s}\right)+(g-1) \wp(x), \\
\frac{\psi^{\prime \prime \prime}}{\psi}=\left[\gamma+\sum_{s=1}^{g-1} \zeta\left(x+\lambda_{s}\right)-(g-1) \zeta(x)\right]^{3}+3\left[\gamma+\sum_{s=1}^{g-1} \zeta\left(x+\lambda_{s}\right)-(g-1) \zeta(x)\right] \\
\times\left[-\sum_{s=1}^{g-1} \wp\left(x+\lambda_{s}\right)+(g-1) \wp(x)\right]-\sum_{s=1}^{g-1} \wp^{\prime}\left(x+\lambda_{s}\right)+(g-1) \wp^{\prime}(x) .
\end{gathered}
$$

Note that the all right-hand sides of these equalities are elliptic functions of the argument $x$. Substitution of these expressions into (4) yields

$$
\begin{aligned}
B(x)= & {\left[\gamma+\sum_{s=1}^{g-1} \zeta\left(x+\lambda_{s}\right)-(g-1) \zeta(x)\right]^{3}+3\left[\gamma+\sum_{s=1}^{g-1} \zeta\left(x+\lambda_{s}\right)-(g-1) \zeta(x)\right] } \\
& \times\left[-\sum_{s=1}^{g-1} \wp\left(x+\lambda_{s}\right)+(g-1) \wp(x)\right]-\sum_{s=1}^{g-1} \wp^{\prime}\left(x+\lambda_{s}\right)+(g-1) \wp^{\prime}(x) \\
& -i h_{1}\left[\left(\gamma+\sum_{s=1}^{g-1} \zeta\left(x+\lambda_{s}\right)-(g-1) \zeta(x)\right)^{2}-\sum_{s=1}^{g-1} \wp\left(x+\lambda_{s}\right)+(g-1) \wp(x)\right] \\
& -\left[h_{2}+3 g(g-1) \wp(x)\right]\left[\gamma+\sum_{s=1}^{g-1} \zeta\left(x+\lambda_{s}\right)-(g-1) \zeta(x)\right] \\
& +i h_{3}+i g(g-1) h_{1} \wp(x)+g(g-1)(g-2) \wp^{\prime}(x)=0 .
\end{aligned}
$$

The function $B(x)$ is elliptic and might have poles up to third order at the points $x=0, x=-\lambda_{s}(s=1, \cdots, g-1)$. However, the direct inspection of the Laurent decompositions near these points shows that all the coefficients at the terms $x^{-3}, x^{-2},\left(x+\lambda_{s}\right)^{-3},\left(x+\lambda_{s}\right)^{-2}$ vanish identically for arbitrary $\gamma$ and $\left\{\lambda_{s}\right\}$. Hence this function can be written in the form

$$
B(x)=b_{0} \zeta(x)+\sum_{s=1}^{g-1} b_{s} \zeta\left(x+\lambda_{s}\right)+\text { const }
$$

where the constant coefficients $b_{0},\left\{b_{s}\right\}$ should obey the relation

$$
b_{0}+\sum_{s=1}^{g-1} b_{s}=0
$$

(statement (III) of par. 20.12 in [6], e.g.). The Laurent decomposition of (7) near the points $x=-\lambda_{s}$ with the use of (6) allows one to find the coefficients $b_{s}$ explicitly. Due to (7), all of them should vanish. This results in the system of $g-1$ transcendental equations to the parameters $\gamma,\left\{\lambda_{s}\right\}$ :

$$
\begin{aligned}
& 3\left[\gamma+\sum_{s \neq k}^{g-1} \zeta\left(\lambda_{s}-\lambda_{k}\right)+(g-1) \zeta\left(\lambda_{k}\right)\right]^{2}-3(g-1)^{2} \wp\left(\lambda_{k}\right)-3 \sum_{s \neq k}^{g-1} \wp\left(\lambda_{s}-\lambda_{k}\right) \\
& -2 i h_{1}\left[\gamma+(g-1) \zeta\left(\lambda_{k}\right)+\sum_{s \neq k}^{g-1} \zeta\left(\lambda_{s}-\lambda_{k}\right)\right]-h_{2}=0, \quad k=1, \cdots, g-1 .
\end{aligned}
$$

It remains only to calculate the constant term in (8). Equivalently, we calculate

$$
\lim _{x \rightarrow 0}\left(B(x)-\frac{b_{0}}{x}\right)
$$


using the Laurent decomposition of (7) near the point $x=0$. After long but straightforward calculations (performed by the MATHEMATICA ${ }^{\circ}$ program), one finds the condition

$$
\begin{aligned}
& {\left[\gamma+\sum_{s=1}^{g-1} \zeta\left(\lambda_{s}\right)\right]^{3}-i h_{1}\left[\gamma+\sum_{s=1}^{g-1} \zeta\left(\lambda_{s}\right)\right]^{2}-h_{2}\left[\gamma+\sum_{s=1}^{g-1} \zeta\left(\lambda_{s}\right)\right]} \\
& +3(2 g-3)\left[\gamma+\sum_{s=1}^{g-1} \zeta\left(\lambda_{s}\right)\right] \sum_{s=1}^{g-1} \wp\left(\lambda_{s}\right)+(3 g-4) \sum_{s=1}^{g-1} \wp^{\prime}\left(\lambda_{s}\right) \\
& -i h_{1}(2 g-3) \sum_{s=1}^{g-1} \wp\left(\lambda_{s}\right)+i h_{3}=0 .
\end{aligned}
$$

The algebraic system (10)-(11) allows one to determine the parameters $\gamma,\left\{\lambda_{s}\right\}$ under which the elliptic function $B$ has no poles and equals zero at one point. Then $B(x)=0$ due to the Liouville theorem (statement (IV) of par. 20.12 in [6]). The last equation is cubic in $\gamma$. This corresponds to three linearly independent solutions to the original Equation (4).

\section{Summary}

Let us summarize our results. We obtained the explicit solutions of the separated Equation (4) at integer couplings $g$ which, in its turn, gives the solution to the three-particle quantum Calogero-Moser problem via the procedure described in [10]. We conjecture that $g$ Equations (10)-(11) determine the $g$ parameters $\gamma, \lambda_{s}(s=1, \cdots, g-1)$ in the generic case at the least. However, it is not clear whether the solution to the above problem in the forms known before [7] [8] can be transformed into the forms with separated variables. As [7] [8] [10], we consider in general singular solutions to the differential Equation (4) leaving aside the right physical boundary conditions which are even not known here [10].

\section{Acknowledgements}

The work was supported by the Votruba-Blokhintsev CR-JINR cooperation program in theoretical physics, Czech Science Foundation project 17-01706S and NPI CAS institutional support RVO 61389005.

\section{References}

[1] Sutherland, B. (1971) Quantum Many-Body Problem in One Dimension: Ground State. Journal of Mathematical Physics, 12, 246-250. https://doi.org/10.1063/1.1665584

[2] Sutherland, B. (1972) Exact Results for a Quantum Many-Body Problem in One Dimension II. Physical Review A, 5, 1372-1376. https://doi.org/10.1103/PhysRevA.5.1372

[3] Olshanetsky, M.A. and Perelomov, A.M. (1983) Quantum Integrable Systems Related to Lie Algebras. Physics Reports, 94, 313-404. https://doi.org/10.1016/0370-1573(83)90018-2

[4] Calogero, F. (1975) Exactly Solvable One-Dimensional Many-Body Problems. Lettere al Nuovo Cimento, 13, 411-416. https://doi.org/10.1007/BF02790495 
[5] Moser, J. (1975) Three Integrable Hamiltonian Systems Connected with Isospectral Deformations. Advances in Mathematics, 16, 197-220.

https://doi.org/10.1016/0001-8708(75)90151-6

[6] Whittaker, E.T. and Watson, G.N. (1927) A Course of Modern Analysis. University Press, Cambridge.

[7] Dittrich, J. and Inozemtsev, V.I. (1993) On the Structure of Eigenvectors of Multidimensional Lamé Operator. Journal of Physics A: Mathematical and General, 26, L753-L756. https://doi.org/10.1088/0305-4470/26/16/008

[8] Felder, G. and Varchenko, A. (1995) Integral Representation of Solutions of the Elliptic Knizhnik-Zamolodchikov-Bernard Equations. International Mathematics Research Notices, 5, 221-233. https://doi.org/10.1155/S1073792895000171

[9] Komori, Y. and Takemura, K. (2002) The Perturbation of the Quantum Calogero-Moser-Sutherland System and Related Results. Communications in Mathematical Physics, 227, 93-118. https://doi.org/10.1007/s002200200622

[10] Sklyanin, E.K. (1995) Separation of Variables: New Trends. Progress of Theoretical Physics Supplement, 118, 35-60. https://doi.org/10.1143/PTPS.118.35 